\title{
Identification of the prosurvival activity of nerve growth factor on cardiac myocytes
}

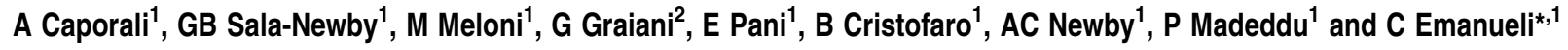

Neurotrophins (NTs) control neuron survival and regeneration. Recent research showed that NTs possess cardiovascular actions. In this study, we investigated the hypothesis that the NT nerve growth factor (NGF) prevents cardiomyocyte apoptosis. We demonstrated that cultured rat neonatal cardiomyocytes (RNCMs) produce NGF and express its trkA (tropomyosin-related receptor A (NGF high-affinity receptor)) receptor. RNCMs given a neutralizing antibody for NGF or the trkA inhibitor K252a underwent apoptosis, thus suggesting that NGF is an endogenous prosurvival factor for cardiomyocytes. Adenovirus (Ad)mediated NGF overexpression protected RNCMs from apoptosis induced by either hypoxia/reoxygenation or angiotensin II (Angll). Similarly, recombinant NGF inhibited Angll-induced apoptosis in isolated rat adult cardiomyocytes. Finally, in a rat model of myocardial infarction, NGF gene transfer promoted cardiomyocyte survival. In RNCMs, recombinant NGF induced trkA phosphorylation, followed by Ser473 phosphorylation and nuclear translocation of phospho-protein kinase B (Akt). In response to Akt activation, Forkhead transcription factors Foxo-3a and Foxo-1 were phosphorylated and excluded from the nucleus. The prosurvival effect of adenoviral vector carrying the human NGF gene was inhibited in vitro by K252a, LY294002 (a panphosphatidyl inositol 3-kinase - PI3K - inhibitor), an Akt small interfering RNA, and adenoviruses carrying a dominant negative mutant form of Akt (Ad.DN.Akt) or an Akt-resistant Foxo-3a (Ad.AAA-Foxo-3a). These results newly demonstrate the cardiac prosurvival action of NGF and provide mechanistic information on the signaling pathway, which encompasses trkA, PI3K-Akt, and Foxo.

Cell Death and Differentiation (2008) 15, 299-311; doi:10.1038/sj.cdd.4402263; published online 9 November 2007

Neurotrophins (NTs) were initially considered for their prosurvival and regenerative actions on the nervous system. These beneficial actions of NTs are mediated by their tropomyosin-related kinase receptors (trks), which are tyrosin kinases. ${ }^{1,2}$ Recent studies have unraveled the potential of nerve growth factor (NGF) and other NTs for vascular biology. ${ }^{3-7}$ Our group discovered that NGF protects endothelial cells and skeletal myocytes from apoptosis and promotes neovascularization in murine ischemic limb muscles and diabetic skin wounds. ${ }^{3,5,6}$ We demonstrated the involvement of protein kinase B (Akt) in NGF-induced angiogenesis. ${ }^{3}$

Although others already tracked the presence of NGF in the heart and isolated cardiomyocytes, ${ }^{8-11}$ the hypothesis that NGF controls cardiomyocyte survival was, to the best of our knowledge, never investigated.

In noncardiovascular cells, NGF, via Akt, promotes the nuclear exclusion of Foxo-3a and Foxo-1, two members of the Forkhead box, class $O$ (Foxo) subfamily of Forkhead transcription factors, which stimulate cell death. ${ }^{12}$ Nuclear localization of Foxo activates several Foxo-responsive genes, including the proapoptotic TNF $\alpha$, Fas ligand, and Bim. ${ }^{13-16}$ Akt-dependent Foxo phosphorylation inactivates the Foxo nuclear translocation signal and promotes the interaction of Foxo with 14-3-3 proteins, thus keeping Foxo out of the nucleus. In the cytosol, Akt-phosphorylated Foxo is targeted for proteasomal degradation. ${ }^{14}$

Interestingly, Skurk et al. ${ }^{17}$ reported the presence of an Akt/ Forkhead signaling axis in cardiomyocytes in vitro and in vivo and Morris et al. ${ }^{18}$ showed that overexpression of Foxo-1 in the cardiomyocyte nucleus activates the transcription of the proapoptotic factor TNF $\alpha$-related apoptosis-inducing ligand (TRAIL).

Here we report the discovery that NGF is an endogenous prosurvival factor for the cardiomyocyte and describe the NGF antiapoptotic pathway. This is initiated by the NGF highaffinity receptor trkA (tropomyosin-related receptor A (NGF high affinity receptor)) and involves the nuclear/cytoplasmatic shuttling of Akt, Foxo-3a, and Foxo-1. Moreover, we demonstrate that NGF gene transfer into the rat infarcted heart prevents cardiomyocyte apoptosis, thus providing evidence that the prosurvival effect of this NT may have a potential for cardiac protection.

\section{Results}

Rat neonatal cardiomyocytes produce NGF and express trkA. It has been already suggested that cardiomyocytes express NGF. ${ }^{9}$ However, the possibility that the

\footnotetext{
${ }^{1}$ The Bristol Heart Institute, University of Bristol, Bristol, UK and ${ }^{2}$ Pathology, University of Parma, Parma, Italy

${ }^{*}$ Corresponding author: C Emanueli, Bristol Heart Institute, University of Bristol, BRI-7, Upper Maudlin Road, Bristol BS2 8HW, UK. Tel: + 4401779283512 ; Fax: + 00440117928 3904; E-mail: costanza.emanueli@bristol.ac.uk or c.emanueli@yahoo.co.uk

Keywords: NGF; cardiomyocytes; TrkA; apoptosis; Akt; Foxo

Abbreviations: Ad.NGF, adenoviral vector carrying the human NGF gene; Ad. $\beta$ Gal, adenoviral vector carrying the beta-galactosidase gene; Akt, protein kinase B; Angll, angiotensin II; CCM, conditioned culture medium; Foxo, Forkhead box, class O (Foxo) subfamily of Forkhead transcription factors; H/R, hypoxia/ reoxygenation; MI, myocardial infarct; NGF, nerve growth factor; NT, neurotrophin; RNCMs, rat neonatal cardiomyocytes; siRNA, small interfering RNA; trkA, tropomyosin-related receptor A (NGF high-affinity receptor); TUNEL, terminal deoxy-nucleotideyltransferase-mediated dUTP nick end-labeling

Received 11.5.07; revised 17.9.07; accepted 01.10.07; Edited by RA Knight; published online 09.11.07
} 
cardiomyocyte may be a target of the NGF action was, to the best of our knowledge, never proposed.

In this study, we confirmed the expression of NGF in rat neonatal cardiomyocytes (RNCMs) by immunocytochemistry (Figure 1a) and RT-PCR (Figure 1b). In addition, by ELISA, we detected NGF in the serum-free conditioned culture media (CCM) of RNCMs (Figure 1d). Moreover, as shown in Figure 1e, the CCM of RNCMs promoted neurite outgrowth from PC12 cells (PC12), an action that is reportedly mediated by NGF. ${ }^{9}$ Consistently, a neutralizing antibody for NGF (Ab-NGF, goat polyclonal, $1 \mu \mathrm{g} / \mathrm{ml})^{3}$ prevented the effect of
CCM on PC12 differentiation. Finally, by RT-PCR and western blot, we showed that RNCMs express the trkA receptor of NGF (Figure $1 b$ and $c$ ). Altogether, these results indicate that RNCMs not only produce and release functionally competent NGF but are also equipped to respond to NGF.

An autocrine mechanism mediates the prosurvival action of NGF. RNCMs reportedly undergo apoptotic cell death following hypoxia/reoxygenation $(H / R)^{19}$ or stimulation with angiotensin II (Angll). ${ }^{20}$ We examined whether stressed RNCMs release NGF for self-protection.

a
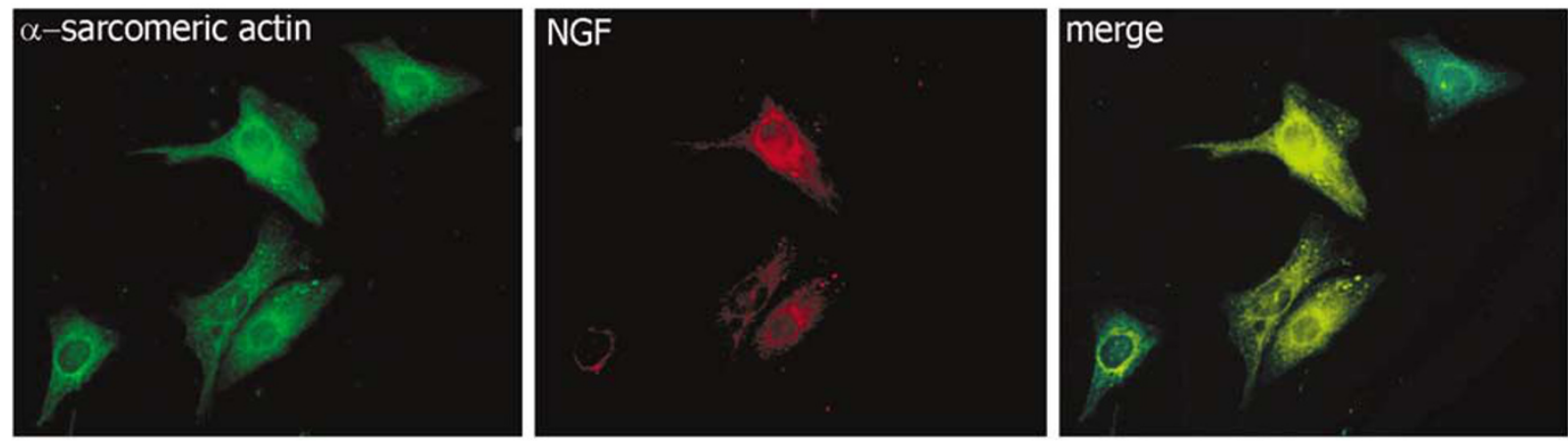

b

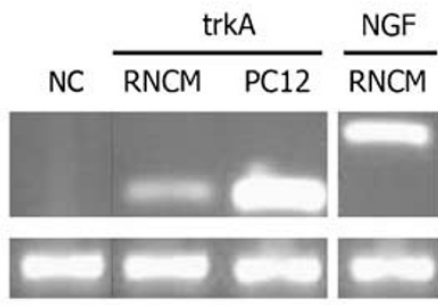

rRNA

$18 S$

d

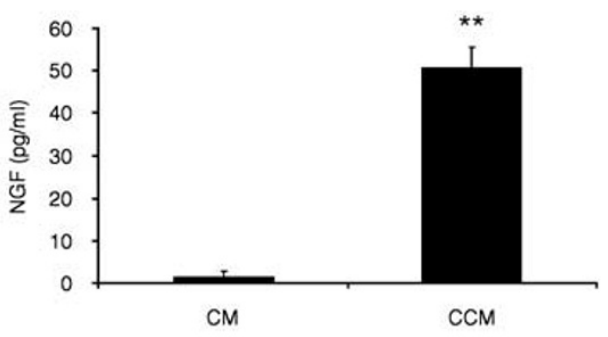

C

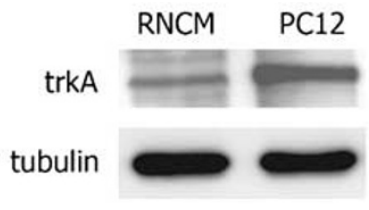

e

$\mathrm{CM}$

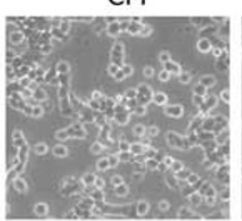

CCM
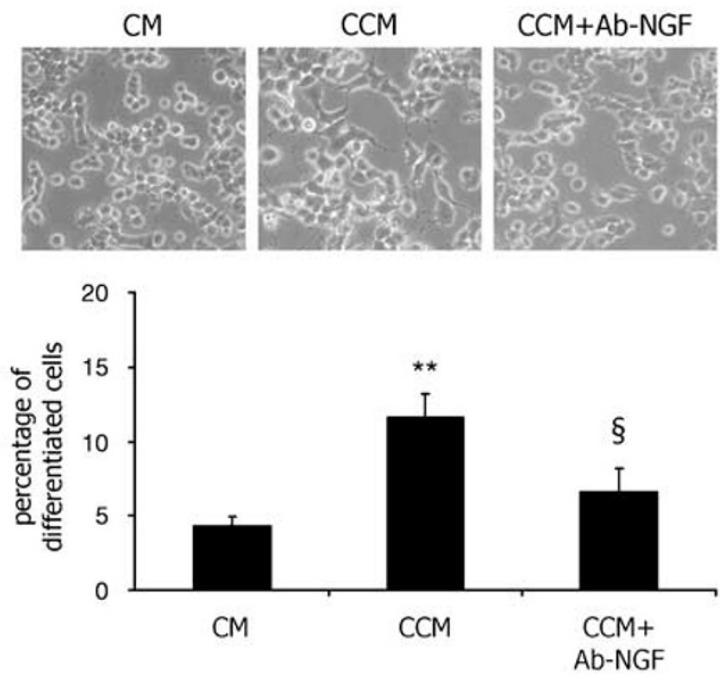

Figure 1 Rat neonatal cardiomyocytes (RNMCs) express NGF and trkA. (a) Immunofluorescence analysis of the cardiac marker $\alpha$-sarcomeric actin (green fluorescence) and NGF (red fluorescence) and merged images (costaining results in yellow fluorescence). (b) RT-PCR analysis of trkA and NGF expression in RNMCs. PC12 cells, which express high level of trkA, were used for reference. Bands are representative of three experiments. Ribosomal RNA (18S) was used as a loading control. Water was used as negative control (NC). (c) Western blot analysis of trkA in lysates of RNCMs. PC12 protein extracts were loaded as positive controls for trkA. Bands are representative of three experiments. (d) Determination of NGF content in the RNCM conditioned culture medium (CCM) and in basal culture medium (CM) by ELISA. Data are presented as mean \pm S.E.M. $(n=3) .{ }^{* *} P<0.01$ versus CM. (e) Secreted NGF from RNCM-mediated differentiation (neurite extension) of PC12 cells. PC12 morphology was observed after incubation for 3 days in the CCM of RNCMs or in CM. A NGF-neutralizing antibody (Ab-NGF) prevented PC12 differentiation. Data are presented as mean \pm S.E.M. $(n=3$ ). ${ }^{* *} P<0.01$ versus $\mathrm{CM} ;{ }^{\circledR} P<0.05$ versus $\mathrm{CCM}$ 
To this aim, serum-free-maintained RNCMs were submitted to $12 \mathrm{~h}$ hypoxia followed by $24 \mathrm{~h}$ reoxygenation $(\mathrm{H} / \mathrm{R})$ or were stimulated with Angll $\left(10^{-7} \mathrm{M}\right)$ for $24 \mathrm{~h}$. As shown by Figure $2 \mathrm{a}$, the content of NGF in the CCM of RNCMs increased under both conditions. We then asked whether endogenous NGF, via trkA, promotes cardiomyocyte survival in an autocrine way. To this aim, serum-free-cultured RNCMs were incubated for $48 \mathrm{~h}$ in the presence of Ab-NGF or $\mathrm{K} 252 \mathrm{a}(100 \mathrm{nM})$, an inhibitor of trkA. ${ }^{21}$ Controls received nonimmune goat serum $(0.1 \%$ in PBS) or $0.1 \%$ DMSO. Blocking experiments were also performed on RNCMs under $\mathrm{H} / \mathrm{R}$ or Angll treatments. To this aim, Ab-NGF, K252a, and the respective controls were added 30 min before starting $\mathrm{H} / \mathrm{R}$ or incubation with Angll. Since caspase activation is implicated in the initiation as well as execution of the apoptotic program triggered by diverse signals, ${ }^{22}$ we stained our culture for cleaved (activated) caspase-3 to detect apoptotic cell death. The percentage of cleaved capase-3-positive apoptotic RNCMs was calculated. As shown in Figure $2 b$ and $c$, blockade of NGF or its receptor led to increased RNCM apoptosis and also enhanced the proapoptotic effect of $\mathrm{H} / \mathrm{R}$ or Angll, thus suggesting the presence of an autocrine NGF-driven signaling, instrumental to cardiomyocyte survival. Results were confirmed by terminal deoxy-nucleotideyltransferase-mediated dUTP nick end-labeling (TUNEL) assay (data not shown).

NGF signaling in RNCMs. In order to identify the prosurvival signaling mechanism initiated by NGF in cardiomyocytes, serum-free-cultured RNCMs were stimulated with recombinant NGF $(50 \mathrm{ng} / \mathrm{ml})$ for 10,20 , or $60 \mathrm{~min}$. As shown in Figure 3a, NGF supported the phosphorylation of trkA (in Tyr490, active form), Akt (in Ser473, active form), Foxo-3a (in Thr32, inactive form), and Foxo-1 (in Ser256, inactive form). The phosphorylation of trkA and Akt peaked at $20 \mathrm{~min}$ from NGF addition and returned to the basal level by $60 \mathrm{~min}$, whereas the phosphorylation of both Foxo-3a and Foxo-1 was maximal at $60 \mathrm{~min}$. In separate experiments, $30 \mathrm{~min}$ before NGF, RNCMs were pretreated with K252a $(100 \mathrm{nM})$, the PI3K inhibitor LY294002 $(50 \mu \mathrm{M})$, or vehicle $(0.1 \%$ DMSO). As shown in Figure 3b, K252a completely prevented Akt phosphorylation, thus suggesting that trkA mediates NGFinduced Akt activation. As shown in Figure 3c, LY294002 prevented the phosphorylation of Akt, Foxo-3a, and Foxo-1 in response to NGF, thus suggesting that NGF controls Foxo through PI3K-Akt.

Next, we investigated whether the Akt/Foxo pathway is activated by endogenous NGF. To this aim, RNCMs were incubated with Ab-NGF or K252a (or the respective controls) for $48 \mathrm{~h}$ in serum-free medium. Cells were then lysated to perform western blot analyses of Ser473-phospho-Akt, Thr32-phospo-Foxo-3a, Ser256-phospho-Foxo-1, and the respective total forms. As shown in Figure 3d, both Ab-NGF and K252a impaired the phosphorylation of Akt, Foxo-3A, and Foxo-1.

NGF induces the nuclear/cytoplasmic shuttling of phospho-Akt and Foxo in RNCMs. We then evaluated the effect of NGF on the intracellular compartmentalization of
Akt and Foxo. Serum-free-cultured RNCMs were given LY294002 or vehicle $30 \mathrm{~min}$ before adding NGF $(50 \mathrm{ng} / \mathrm{ml})$ or PBS control. After $20 \mathrm{~min}$, RNCMs were fixed and stained for phospho-Akt, total Foxo-3a, or total Foxo-1. As illustrated in Figure $4 \mathrm{a}$, under conditions of serum starvation, control RNCMs showed predominant nuclear localization of Foxo-3a and Foxo-1, while phospho-Akt was mainly expressed in the cytosol. NGF-stimulated RNCMs showed an inverse pattern, with phospho-Akt being mainly located in the nucleus and Foxo-3a and Foxo-1 in the cytosol. PI3K inhibition by LY294002 prevented NGF-induced effect on Akt and Foxo localization. The finding that NGF promotes the nuclear accumulation of active phospho-Akt and the nuclear exclusion of both Foxo-3A and Foxo-1 was confirmed by western blot analyses on cytosolic and nuclear fractions (Figure 4b).

Ad.NGF increases the phosphorylation of Akt, Foxo-3a, and Foxo-1. We then evaluated the effect of prolonged NGF overexpression, which was achieved by means of adenovirus $(A d)$-mediated NGF gene transfer, on Akt and Foxo phosphorylation. Successful adenovirus-mediated transduction of RNCMs using adenoviral vector carrying the human NGF gene (Ad.NGF) (carrying a V5-tag, 50 M.O.I.) was confirmed by RT-PCR, using primers that specifically amplify human but not rat NGF cDNA (Supplementary Figure S1a). Transgene expression was not affected by either $H / R$, or incubation with Angll (Supplementary Figure S1a). Furthermore, western blot analyses using an antibody antiV5 tag performed on both RNCM cell lysates and the RNCM serum-free CCM confirmed successful transgene expression (Supplementary Figure S1b). Neither H/R, nor Angll interfered with transgene expression at the protein level (Supplementary Figure S1b).

Next, by ELISA, we measured 47-fold higher concentration of NGF in the CCM of NGF-transduced RNCMs, as compared with the CCM of $\beta$-Gal-transduced RNCMs (Supplementary Figure S1c). NGF gene transfer promoted phosphorylation of Akt, Foxo-1, and Foxo-3a in cardiomyocytes (Supplementary Figure S1d). This pattern of phosphorylation is consistent with that observed after stimulation with recombinant NGF.

NGF gene transfer inhibits apoptosis of RNCMs. In order to demonstrate that NGF strengthens the resistance of cardiomyocytes to stress, we performed two different apoptotic assays on cultured RNCMs. Both cleaved (activated) caspase-3 staining (Figure 5a) and TUNEL assay (Supplementary Figure S2a) of PBS-treated wells showed that the number of apoptotic RNCMs increases following $\mathrm{H} / \mathrm{R}$ and that Ad.NGF reduces $\mathrm{H} / \mathrm{R}$-induced apoptosis. We additionally examined the effect of Ad.NGF on Angll-induced apoptosis. To this aim, RNCMs were incubated with Angll $\left(10^{-7} \mathrm{M}\right)$ for $24 \mathrm{~h}$ before determining apoptosis by both cleaved caspase-3 staining (Figure $5 \mathrm{~b}$ ) and TUNEL assay (Supplementary Figure S2b). In both cases, we found that Ad.NGF protects cardiomyocytes from Angll-induced apoptosis.

Mechanism of NGF-induced RNCM survival. We then asked whether the trkA/PI3K/Akt/Foxo pathway is 
a

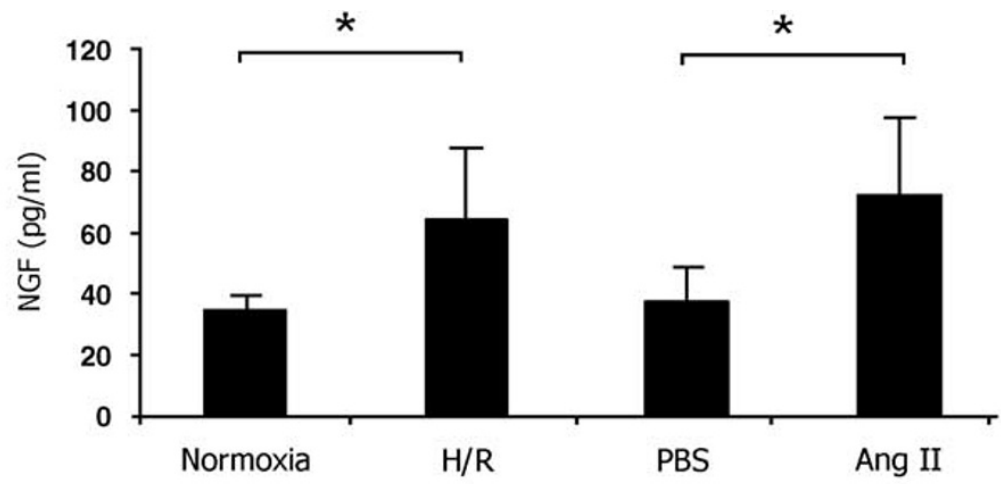

b

$0.1 \%$ goat IGg

Ab-NGF

K252a
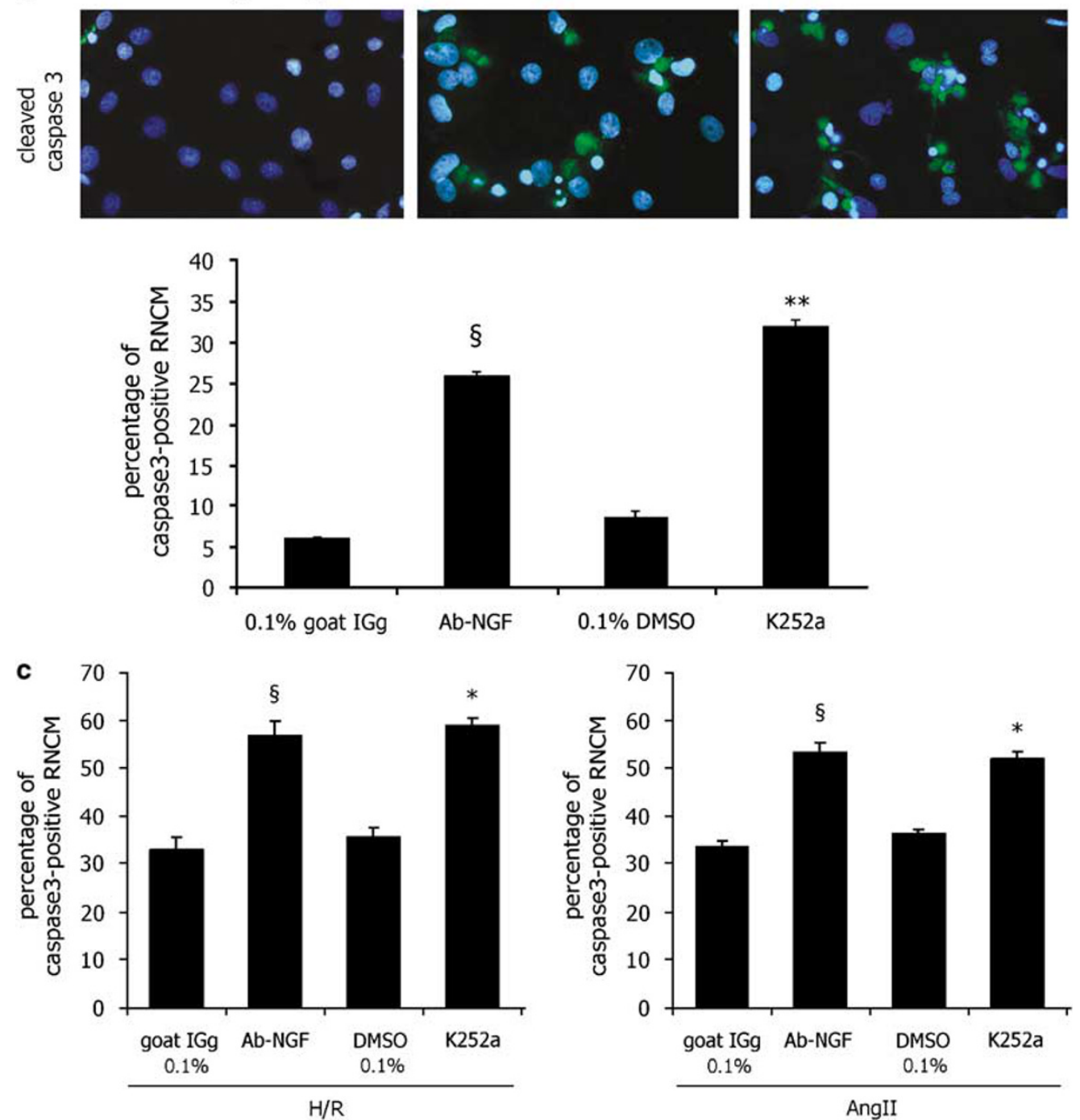

AngII

Figure 2 The release of NGF from RNCMs increases under proapoptotic conditions and NGF is an endogenous prosurvival factor for RNCMs. (a) NGF content in the serum-free culture of RNCMs exposed to hypoxia/reoxygenation (H/R, control: normoxia) or angiotensin II (Angll, control: PBS) was measured by ELISA. Respective controls were the serum-free conditioned medium of normoxic RNCMs and PBS-treated RNCMs, respectively. Data are presented as mean \pm S.E.M. $(n=3)$. ${ }^{*} P<0.05$ versus the respective control. (b) RNCMs were incubated for $48 \mathrm{~h}$ in serum-free medium with Ab-NGF (raised in goat) or the trkA inhibitor K252a. Controls consisted of non-immune goat serum $(0.1 \%$ in PBS) or $0.1 \%$ DMSO, respectively. Apoptosis was detected by cleaved caspase-3 immuno-staining (green fluorescence). Nuclei were counterstained by DAPI (blue fluorescence). The fluorescent images $(\times 400)$ are representative of the experiment. Bar graph shows the percentage of cleaved caspase 3-positive RNCMs. Data are presented as mean \pm S.E.M. $(n=3)$. ${ }^{\S} P<0.05$ versus $0.1 \%$ goat lgG; ${ }^{* *} P<0.01$ versus $0.1 \%$ DMSO. (c) RNCMs were submitted to $\mathrm{H} / \mathrm{R}$ or incubated with Angll (for both as described in (a)) in the presence of Ab-NGF, K252a, or the respective controls. Apoptosis was evaluated by cleaved caspase-3 staining, as described in (b). Data are presented as mean \pm S.E.M. $(n=3) .{ }^{\circledR} P<0.05$ versus $0.1 \%$ goat lgG; ${ }^{*} P<0.05$ versus $0.1 \%$ DMSO 
a

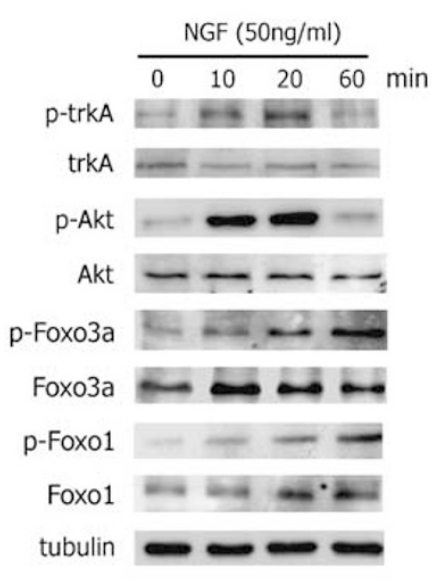

C

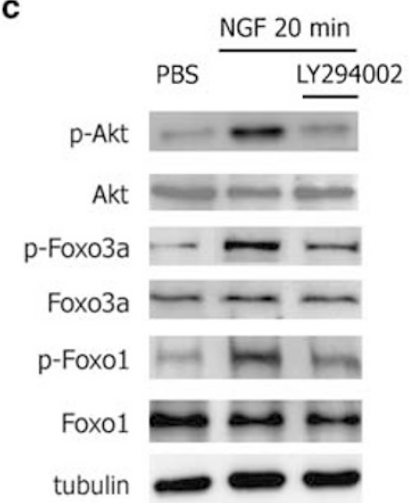

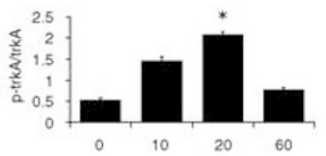
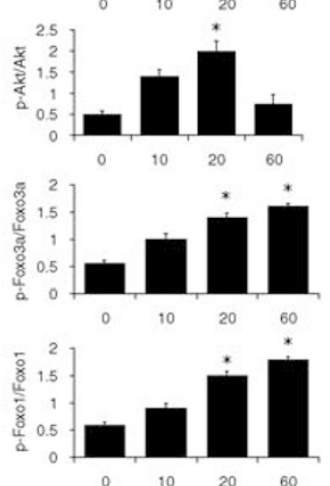

b

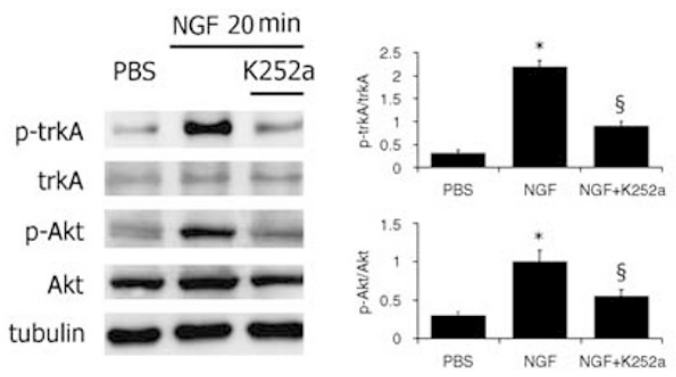

d

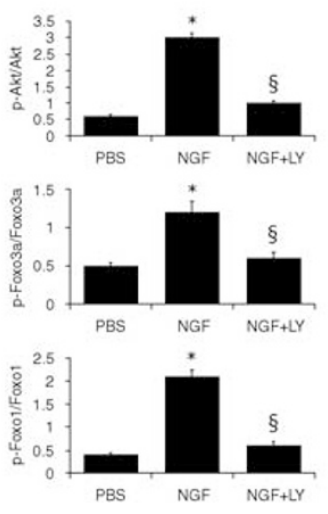

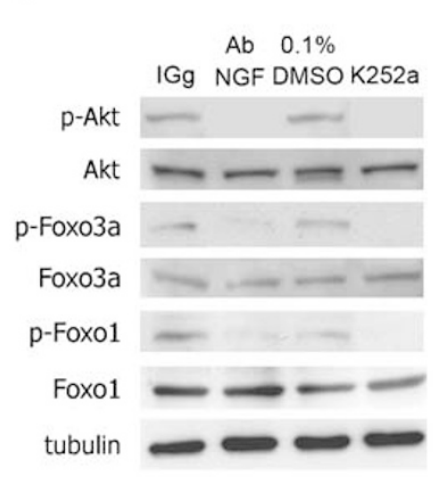

Figure 3 NGF signaling in RNMCs. (a) Serum-starved RNCMs received NGF (50 ng/ml). After 0, 10, 20, and 60 min, the phosphorylation of trkA (Tyr490), Akt (Ser473), Foxo-3a (Thr32), and Foxo-1 (Ser256) was determined by western blot. Tubulin was used as loading control. Graphs show the ratio between the densitometric reading of phosphorylated and total trkA, Akt, Foxo-3a, and Foxo-1. Data are presented as mean \pm S.E.M. $(n=3)$. ${ }^{*} P<0.05$ versus time zero. (b) Serum-starved RNCMs were pretreated with the trkA inhibitor K252a ( $100 \mathrm{nM})$ or its vehicle $(0.1 \%$ DMSO) before adding NGF. After 20 min, the phosphorylation of trkA and Akt was examined. Controls (PBS) received $0.1 \%$ DMSO (instead of K252a) and PBS (instead of NGF). Tubulin was used as loading control. K252a blocked the phosphorylation of both trkA and Akt. Graphs show the ratio between the densitometric reading of phosphorylated and total trkA and phosphorylated and total Akt. Data are presented as mean \pm S.E.M. $(n=3)$. ${ }^{*} P<0.05$ versus PBS; ${ }^{\S} P<0.05$ versus NGF $+0.1 \%$ DMSO (NGF). (c) Serum-starved RNMCs were pretreated with the PI3K inhibitor LY294002 (50 $\mu$ M) or its vehicle $(0.1 \%$ DMSO) before adding NGF. After 20 min, the phosphorylation of Akt, Foxo-3a, and Foxo-1 was evaluated. Controls received $0.1 \%$ DMSO and PBS. Tubulin was used as loading control. LY294002 blocked the phosphorylation of Akt, Foxo-3a, and Foxo-1. Graphs show the ratio between the densitometric reading of phosphorylated and total Akt, phosphorylated and total Foxo-3a, and phosphorylated and total Foxo-1. Data are presented as mean \pm S.E.M. $(n=3) .{ }^{*} P<0.05$ versus PBS; ${ }^{\circledR} P<0.05$ versus NGF $+0.1 \%$ DMSO (NGF). (d) Serum-free-cultured RNCMs were treated for $48 \mathrm{~h}$ with Ab-NGF or K252a. Controls received $0.1 \%$ nonimmune goat serum or $0.1 \%$ DMSO. Tubulin was used as loading control. Either Ab-NGF or K252a reduced phosphorylation of Akt, Foxo-3a, and Foxo-1. Graphs show the ratio between the densitometric reading of phosphorylated and total Akt, phosphorylated and total Foxo-3a, and phosphorylated and total Foxo-1. Data are presented as mean \pm S.E.M. $(n=3) .{ }^{*} P<0.05$ versus $0.1 \%$ goat lgG; ${ }^{\$} P<0.01$ versus $0.1 \%$ DMSO

responsible for the protective effect of Ad.NGF in the H/R test. In particular, we investigated whether trkA blockade by K252a, PI3K inhibition by LY294002, Akt inhibition by Ad.DN-Akt, or Ad.AAA-Foxo-3a-induced overexpression of a constitutive active Foxo-3a (which is protected from Akt phosphorylation) impairs the antiapoptotic action of Ad.NGF on RNCMs. For these experiments, we modified the $H / R$ protocol by shortening hypoxia to $5 \mathrm{~h}$. This was decided because blockade of trkA or PI3K/Akt as well as increased active Foxo-3a may exacerbate $\mathrm{H} / \mathrm{R}$-induced cardiomyocyte death. Notably, the prosurvival action of Ad.NGF was nullified by K252a or LY294002 pretreatment (Figure $6 \mathrm{~b}$ and Supplementary Figure S3b). Moreover, the protective effect of Ad.NGF was blocked by coinfection of RNCMs with either Ad.DN.Akt or Ad.AAA-Foxo-3a (Figure $6 \mathrm{~d}$ and Supplementary Figure S3d). For reference, a set of experiments was also performed on normoxic cells (see Figure $6 a$ and $c$ and Supplementary Figures S3a and S3c). The importance of Akt for NGF prosurvival action was further tested by using an Akt small interfering RNA (Akt siRNA). RNCMs were transfected with Akt siRNA or scramble siRNA (control) overnight. Effective downregulation of Akt protein by Akt siRNA was detected after $48 \mathrm{~h}$ by western blot for Akt (Supplementary Figure S4a). Following transfection with $A k t$ SiRNA or scramble siRNA, RNMCs were transduced with Ad.NGF or adenoviral vector carrying the beta-galactosidase 
a
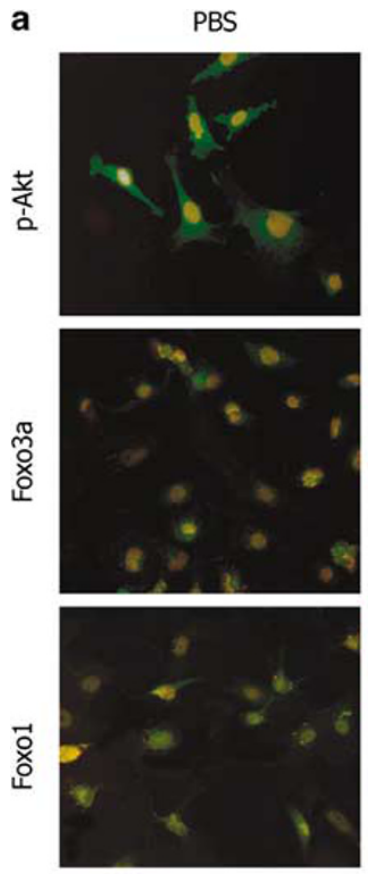

b
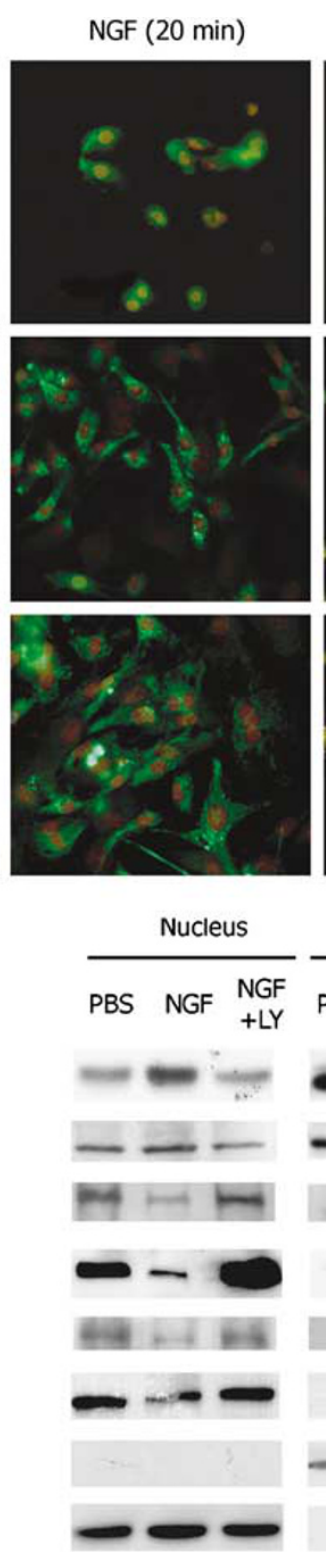

NGF+LY294002
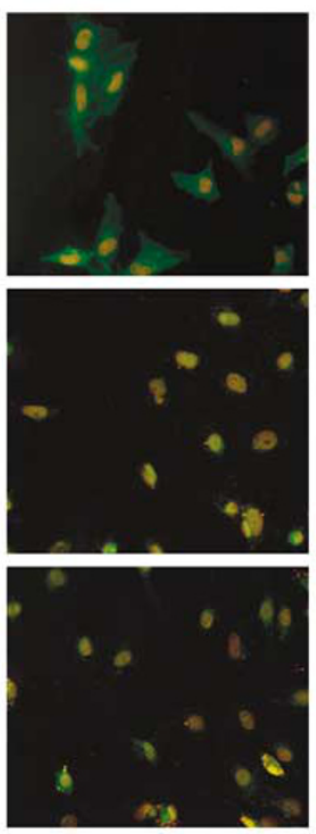

Cytoplasm
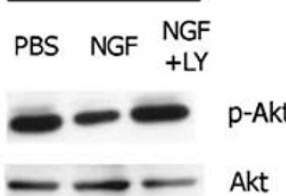

Akt

p-Foxo3a

Foxo3a

p-Foxo1

Foxo1

$\alpha$-sarcomeric actin

Histone $\mathrm{H} 3$
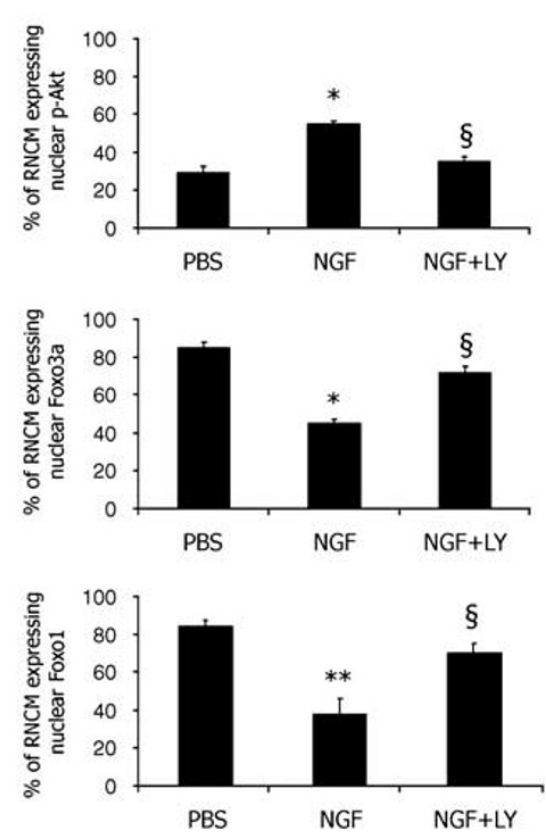

Figure 4 NGF induces nuclear/cytoplasmatic shuttling of phospho-Akt and Foxo-3a and Foxo-1 in RNCMs. (a) Serum-starved RNCMs were pretreated with LY294002 or its vehicle $(0.1 \%$ DMSO) before receiving NGF $(50 \mathrm{ng} / \mathrm{ml})$ or PBS. After $20 \mathrm{~min}$, cells were fixed and stained for phospho-Akt, total Foxo-3a, or total Foxo-1 by using FITCconjugated antibodies (green fluorescence). Nuclei were stained by PI (red fluorescence). Exposure was optimized to demonstrate localization and it does not reflect the concentration of the antigen. NGF induced nuclear localization of phospho-Akt (as demonstrated by the yellow/orange colors of nuclei, which indicates colocalization of FITC and PI) and promoted nuclear export of Foxo-3a and Foxo-1. LY294002 abolished these NGF effects. Graphs show the percentage of RNCMs expressing nuclear phosphoAkt, Foxo-3a, and Foxo-1. Results are from three independent experiments. Data are presented as mean \pm S.E.M. $(n=3)$. ${ }^{*} P<0.05$ and ${ }^{* *} P<0.01$ versus PBS; ${ }^{\circledR} P<0.05$ versus NGF $+0.1 \%$ DMSO (NGF). (b) Serum-starved RNCMs were pretreated with LY294002 or its vehicle $(0.1 \% \mathrm{DMSO})$ for $30 \mathrm{~min}$ before receiving NGF ( $50 \mathrm{ng} / \mathrm{ml})$ or PBS. After $20 \mathrm{~min}$, cells were lysated and nuclear and cytoplasmatic fraction prepared. Nuclear and cytoplasmatic extracts were assessed by western blot using antibodies targeting phospho- and total form of Akt, Foxo-3a, and Foxo-1. Efficient separation of nuclear and cytoplasmatic fractions was confirmed by western blot for histone H3 and $\alpha$-sarcomeric actin, respectively

gene (Ad. $\beta G a)$. Western blot analysis for NGF of Ad.NGFinfected RNCMs showed no difference between Akt siRNA and scramble siRNA (data not shown), thus discounting the possibility that transfection with $\operatorname{siRNA}$ may impair the efficiency of adenovirus-mediated NGF infection. Both immunocytochemistry for cleaved caspase-3 and TUNEL assay showed that $A k t$ siRNA promotes apoptosis under both normoxia and $\mathrm{H} / \mathrm{R}$ and impairs Ad.NGF-induced survival (Supplementary Figures S4b and S4c).
NGF inhibits Angll-mediated apoptosis of rat adult myocytes. Next, we tested whether the antiapoptotic action of NGF expands to adult cardiomyocytes. Cardiac myocytes were extracted from the heart of adult Wistar rat and treated with Angll $\left(10^{-9} \mathrm{M}\right)$ for $24 \mathrm{~h}$ (according to Kajstura et al. $)^{23}$ or submitted to $6 \mathrm{~h}$ hypoxia/18 $\mathrm{h}$ reoxygenation (according to Kang et al.) ${ }^{24}$ in the presence of recombinant NGF $(50 \mathrm{ng} / \mathrm{ml})$ or PBS. TUNEL assay showed that NGF partially prevented apoptosis induced by 
a

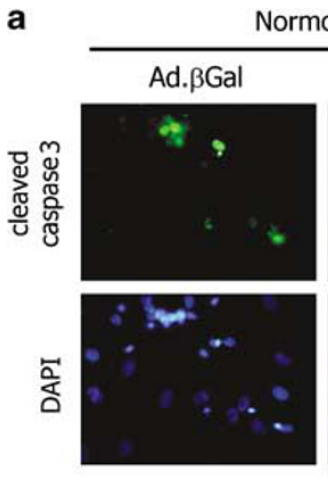

ormoxia

\section{Ad.NGF}
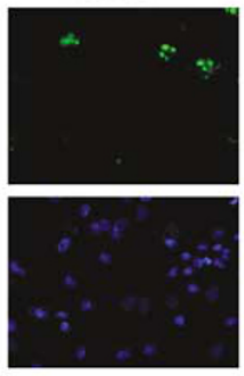

Hypoxia/Reoxygenation
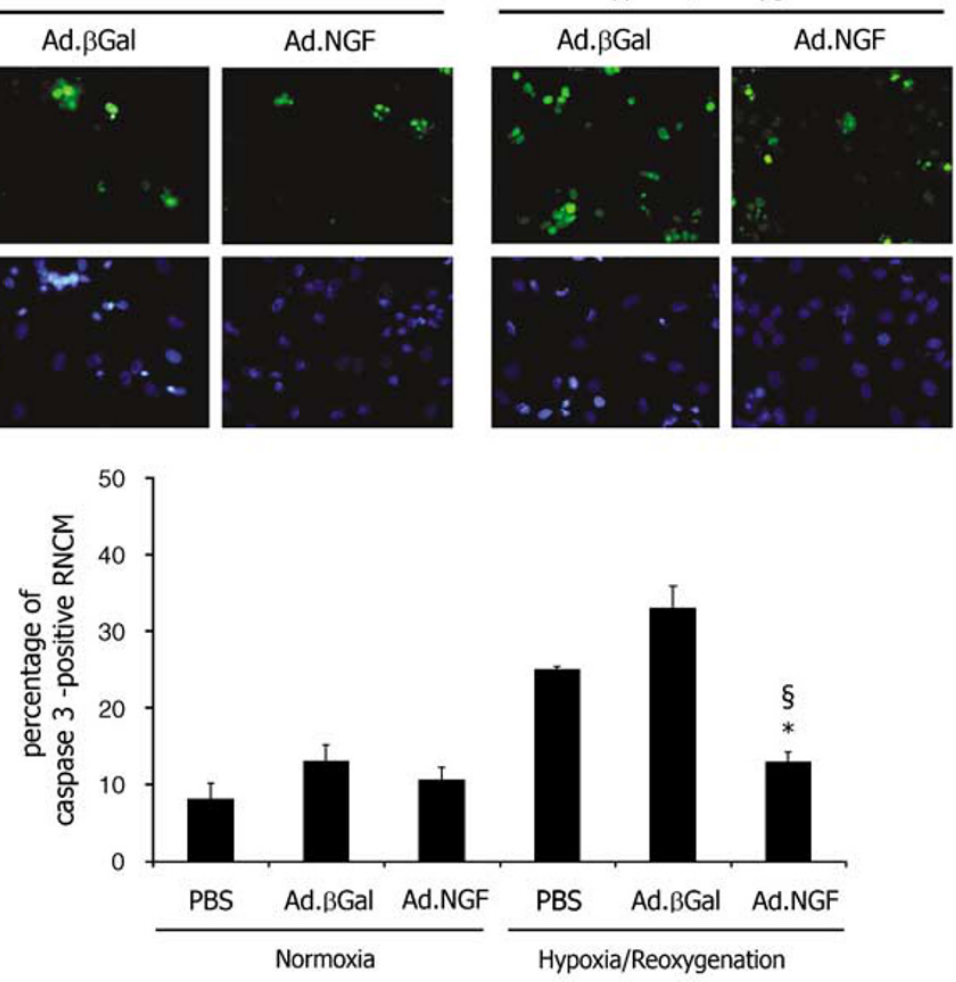

b
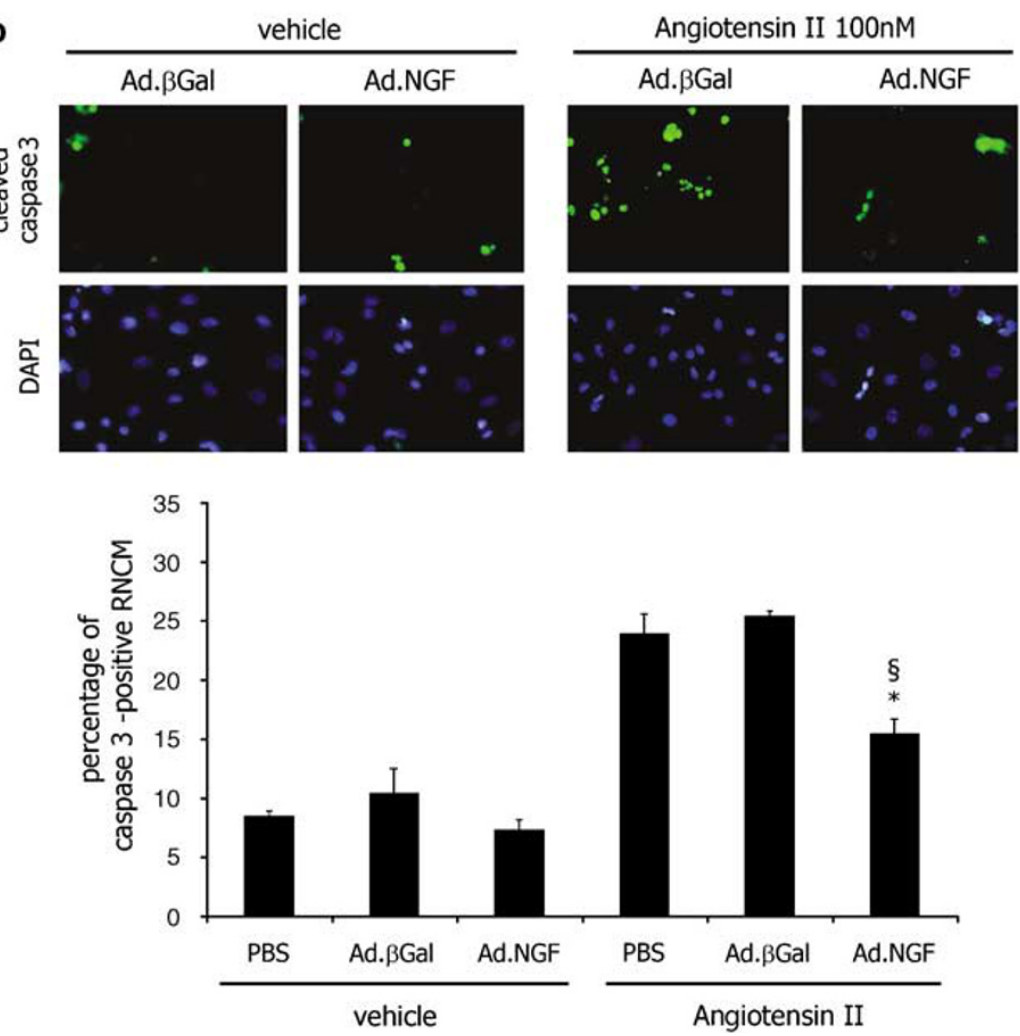

Figure 5 NGF overexpression prevents apoptosis of RNCMs. RNCMs were transduced with Ad.NGF or Ad. $\beta$ Gal, or not infected (PBS). (a) RNCMs underwent $12 \mathrm{~h}$ of hypoxia followed by $24 \mathrm{~h}$ reoxygenation or were maintained under normoxia. (b) RNCMs were infected with $A d$.NGF or $A d$. $\beta$ Gal or given PBS before being stimulated with Angll $\left(10^{-7} \mathrm{M}\right.$, for $24 \mathrm{~h}$ ). Apoptotic RNCMs were detected by cleaved caspase-3 staining (green fluorescence). Bar graphs show the quantitative analysis of apoptosis, which is expressed as percentage of cleaved caspase-3-positive RNCMs. Data are presented as mean \pm S.E.M. $(n=3)$. ${ }^{*} P<0.05$ versus PBS; ${ }^{\circledR} P<0.05$ versus $A d$. $\beta G a l$. Representative images are presented above graphs 
Normoxia
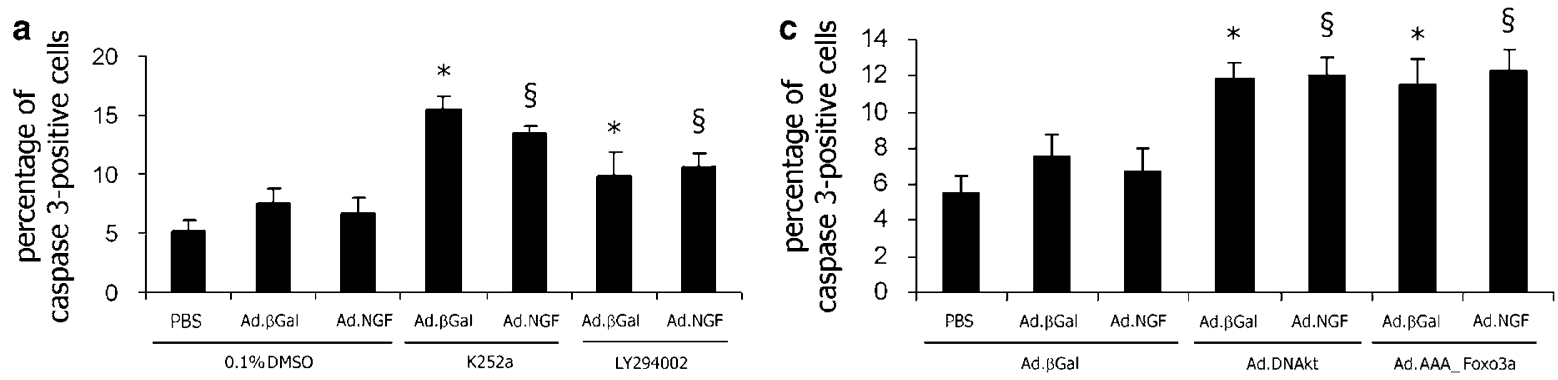

Hypoxia/Reoxygenation
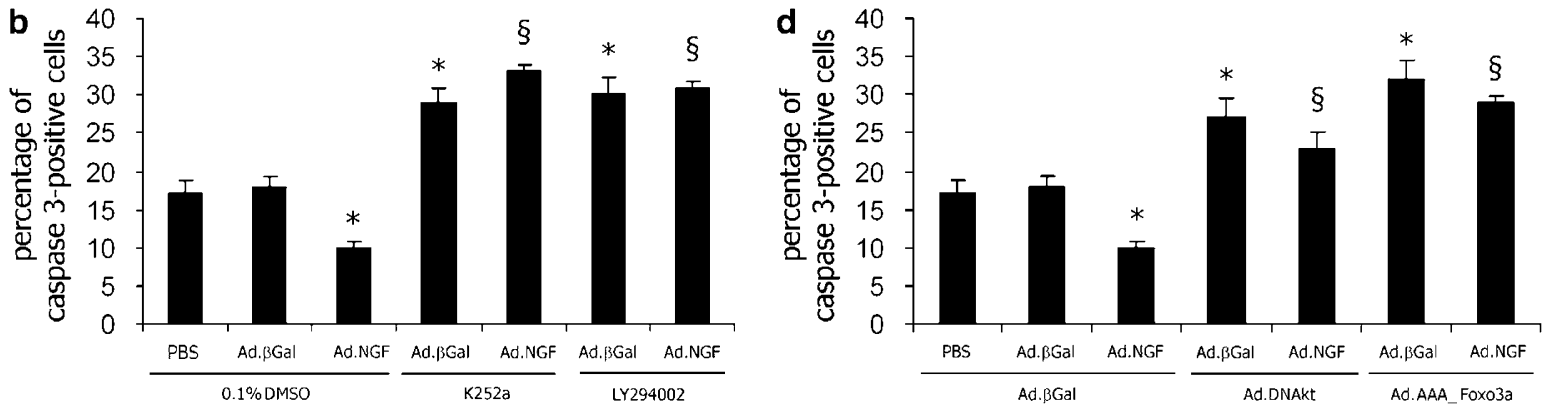

Figure 6 NGF overexpression prevents apoptosis of RNCMs through trkA, Akt, and Foxo-3a. (a, b) RNCMs were infected with Ad.NGF or Ad $\beta$ Gal and then given K252a, LY294002, or $0.1 \%$ DMSO (vehicle). Not infected RNCMs were given PBS and $0.1 \%$ DMSO were used for reference. (c, d) RNCMs were coinfected with $A d . N G F$ or $A d$. $\beta G a l$ (as control of Ad.NGF) and with an adenovirus carrying a dominant negative mutant form of Akt (Ad.DN-Akt), an adenovirus carrying a constitutionally active form of Foxo-3a (Ad.AAA-Foxo-3a), or Ad. $\beta$ Gal (as control of both Ad.DN-Akt and Ad.AAA-Foxo-3a. RNCMs infected with Ad. $\beta$ Gal and given PBS were used for reference. RNCMs in (a) and (c) were maintained under normoxia. RNCMs in (b) and (d) underwent $5 \mathrm{~h}$ hypoxia and $24 \mathrm{~h}$ reoxygenation. Apoptosis was revealed by cleaved caspase-3 staining. Graphs show the quantitative analysis of apoptosis, which is expressed as percentage of cleaved caspase-3-positive RNCMs. Data are presented as mean \pm S.E.M. $(n=3)$. (a, $\mathbf{b})$ : ${ }^{*} P<0.05$ versus $A d$. $\beta$ Gal plus $0.1 \%$ DMSO; ${ }^{\S} P<0.05$ versus $A d . N G F$ plus $0.1 \%$ DMSO. (c, d): ${ }^{*} P<0.05$ versus $A d . \beta G a l$ plus $A d$. $\beta$ Gal; ${ }^{\S} P<0.05$ versus $A d . N G F$ plus Ad. $\beta \mathrm{Gal}$

Angll or $\mathrm{H} / \mathrm{R}$ (Figure $7 \mathrm{a}$ and $\mathrm{b}$ ). Similar results were obtained when apoptosis was tracked by cleaved-caspase 3 immunocytochemistry (Supplementary Figures S5a and $\mathrm{S5b})$.

Intra-myocardial NGF gene transfer reduces myocyte apoptosis in the rat infarcted heart. Myocardial infarction (MI) triggers cardiomyocyte apoptosis. Following an immediate massive apoptosis response to coronary occlusion, later cardiomyocyte apoptosis manifests in the peri-infarct myocardium and eventually contributes to maladaptative post-MI ventricular remodeling and progression towards heart failure. ${ }^{25-28}$ We further investigated the prosurvival role of NGF in a rat model of $\mathrm{Ml}$ induced by permanent coronary ligation. To this specific aim, we injected Ad.NGF or the control Ad. $\beta$ Gal in the periinfarct myocardium. Successful gene transfer was confirmed (at 3 days post-MI and gene delivery) by RT-PCR using primers that specifically amplify human but not rat NGF cDNA (data not shown). Moreover, at 7 days from gene delivery, transverse sections of the left ventricle were prepared from diastole-arrested and perfusion/fixed hearts. Immunohistochemistry for V5 showed the presence of V5 in the injected myocardium (Supplementary Figure S6). Only a fraction of cardiomyocytes stained positive for V5. However, it must be considered that NGF is secreted to act extracellularly on its trkA receptor. Therefore, nontransduced cardiomyocytes could still benefit of transgenic NGF. Moreover, transgene expression may be higher at earlier time points. Having validated the gene transfer procedure, we next examined the effect of intramyocardial Ad.NGF on cardiomyocyte apoptosis. On sections cut from the same hearts of above, apoptotic cardiomyocytes were revealed by double staining for TUNEL and the specific cardiac marker $\alpha$-sarcomeric actin and counted in the peri-infarct myocardium. As shown by Figure 8, Ad.NGF reduced the percentage of TUNELpositive apoptotic cardiomyocytes $\left({ }^{\star} P<0.05\right.$ versus Ad. $\beta G a l)$.

\section{Discussion}

NTs were christened in consideration of their actions on the nervous system and, for a long time, they were exclusive interest of neuroscientists. This family of polypeptides was known to promote the survival and regeneration of neurons, but the possibility that the cardiac muscle may be a direct target of NTs was never investigated before. In fact, even those scientists who found NGF expressed by the myocardium interpreted their findings as a modulation by the cardiomyocyte of its innervation, ${ }^{9}$ while ignoring the possibility 


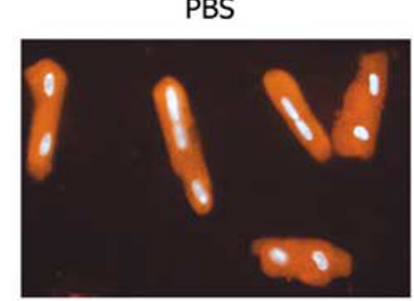

$\mathrm{H} / \mathrm{R}$

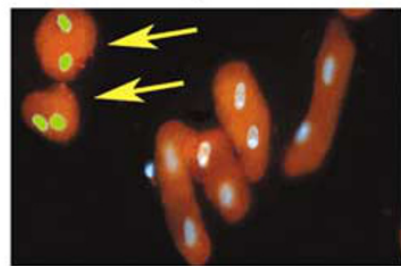

$\mathrm{NGF}+\mathrm{H} / \mathrm{R}$
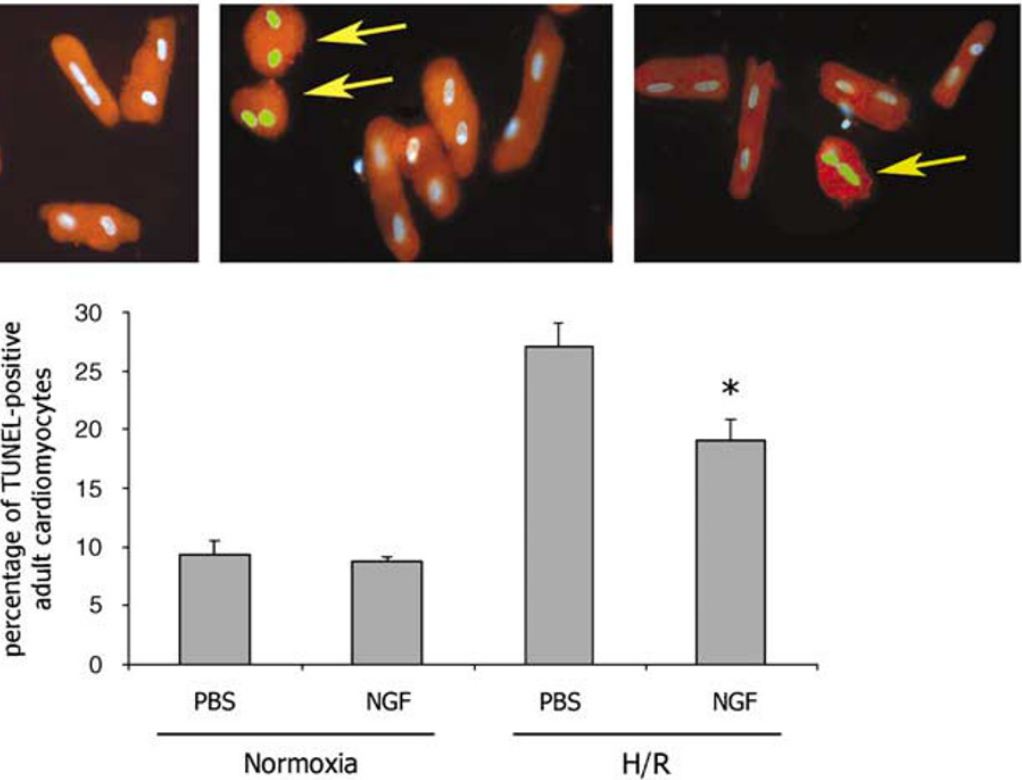

b
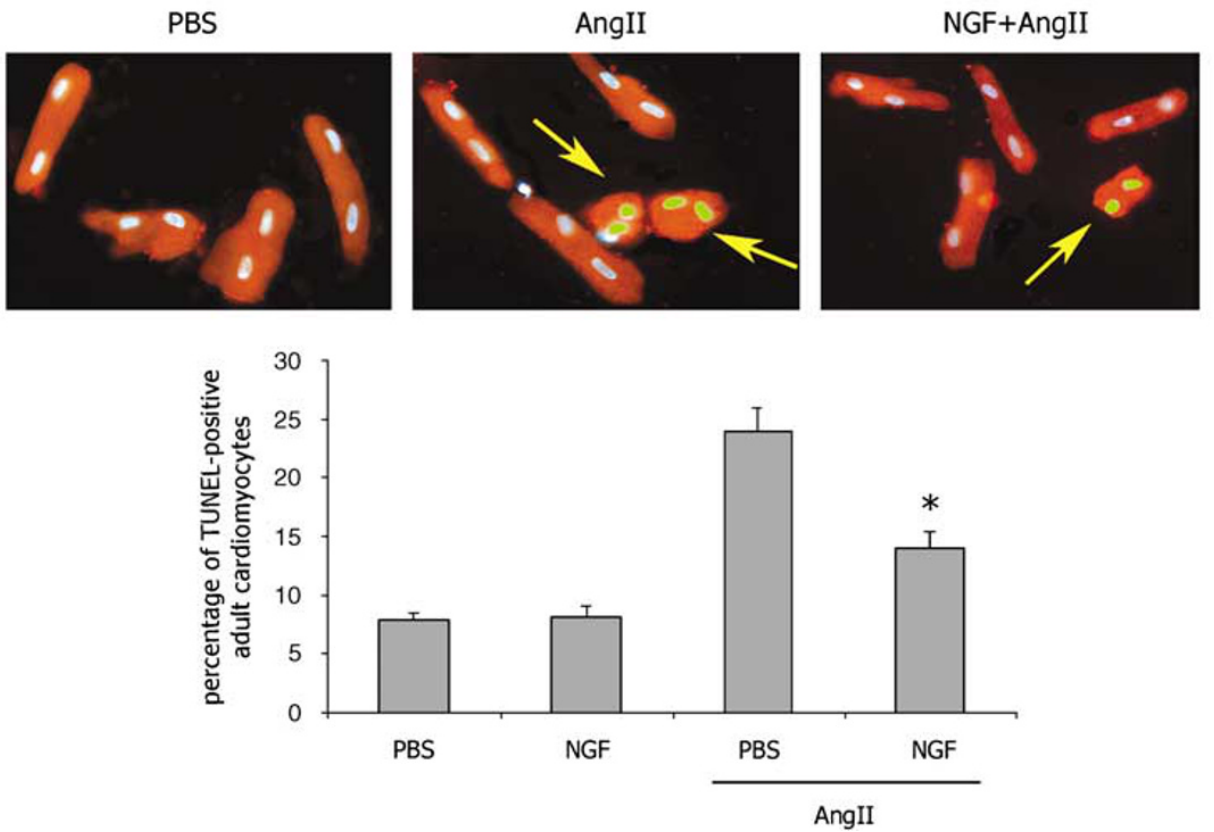

Figure 7 NGF inhibits apoptosis in rat adult cardiomycytes. (a) Isolated rat adult cardiomyocytes were maintained under normoxia or submitted to $6 \mathrm{~h}$ hypoxia followed by $18 \mathrm{~h}$ reoxygenation and cotreated with NGF $(50 \mathrm{ng} / \mathrm{ml})$ or its vehicle PBS. (b) Rat adult cardiomyocytes were incubated with Angll $\left(10^{-9} \mathrm{M}\right)$ or its vehicle PBS and cotreated with NGF $(50 \mathrm{ng} / \mathrm{ml})$ or its vehicle PBS for $24 \mathrm{~h}$. Apoptotic nuclei were identified by TUNEL staining (green fluorescence). $\alpha$-Sarcomeric actin stains cardiomyocytes (red fluorescence). The pictures were captured at $\times 400$ magnification. Arrows point to TUNEL-positive cardiomyocytes. Bar graphs quantify apoptosis, which is expressed as percentage of TUNEL-positive cardiomyocyte. Data are presented as mean \pm S.E.M. $(n=3)$. (a): ${ }^{*} P<0.05$ versus PBS plus H/R; (b): ${ }^{*} P<0.05$ versus PBS plus Angll

that cardiomyocytes could release NGF in response to and for their own protection from stress.

During the last 10 years, the relevance of NTs in the regulation of cardiovascular function has been demonstrated by us and others, including the capacity of NGF to promote angiogenesis and prevent endothelial cell apoptosis. ${ }^{3-6}$

Hereby, we report for the fist time that NGF posses a direct antiapoptotic effect on the cardiomyocyte and describe the underlying signaling mechanism. These results were obtained using different experimental models, which expand from cultured rat neonatal and adult cardiomyocytes to an in vivo rat model of myocardial infarction.

We identified the prosurvival pathway of NGF by working on cultured RNCMs. We found that those cells produce and release NGF in a stress-regulated manner and express the trkA receptor, which binds NGF with high affinity. Upon addition of recombinant NGF to RNCMs, trkA is phosphorylated and, via PI3K, it activates Akt. Active phospho-Akt 

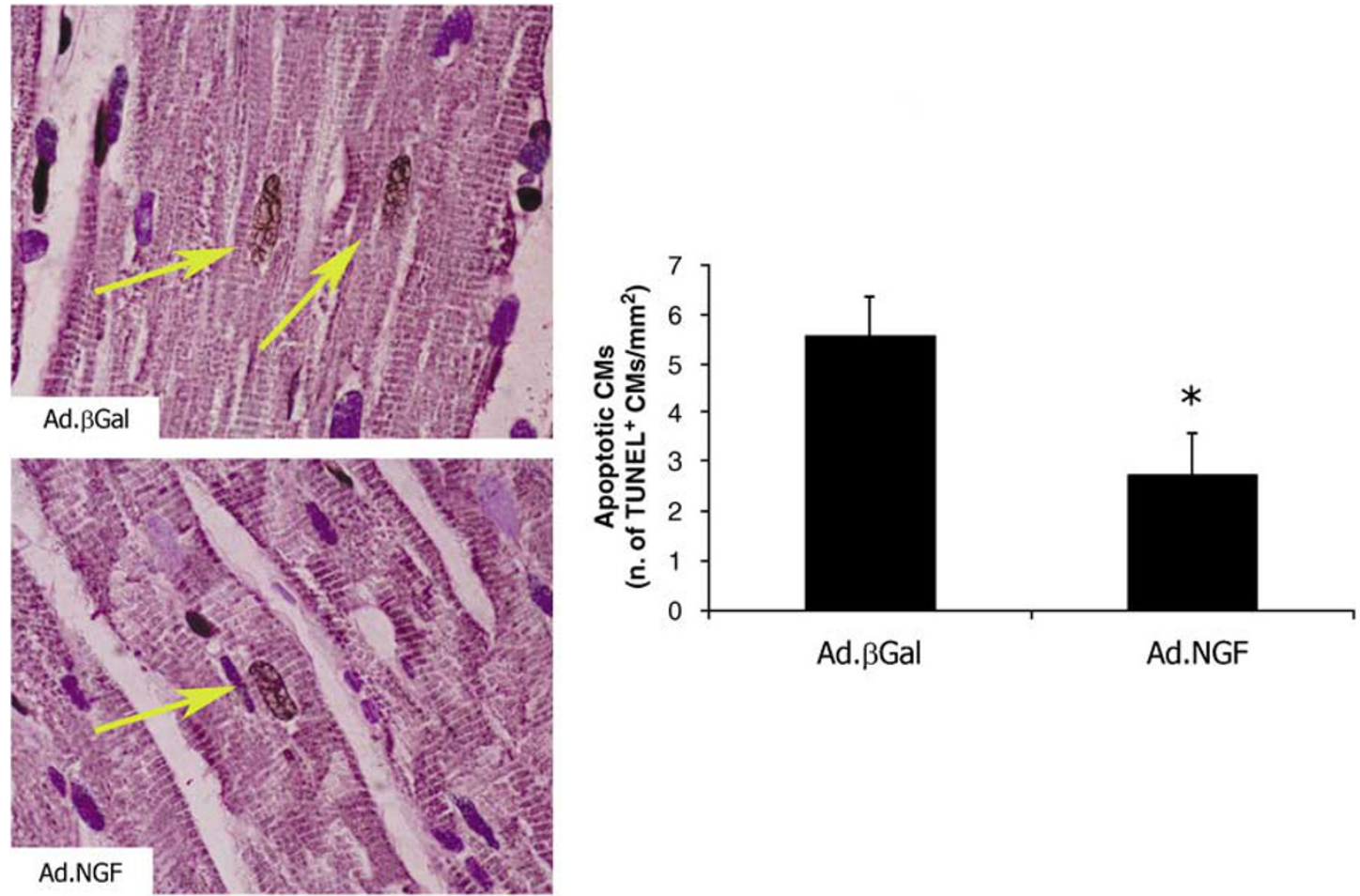

Figure 8 Local NGF gene transfer prevents apoptosis of cardiomyocytes in the rat infarcted heart. Myocardial infarction (MI) was induced in adult Wistar rat. Ad.NGF or Ad. $\beta$ Gal (each at $10^{8}$ p.f.u.) was injected in the peri-infarct myocardium. After 7 days, the heart was arrested in diastole and perfusion/fixed. Apoptosis of cardiomyocytes (CMs) was revealed by double staining for TUNEL (TUNEL-positive nuclei are stained in dark brown) and the cardiac marker $\alpha$-sarcomeric actin (in purple). Nuclei were counterstained with hematoxylin. In the pictures captured (optical microscopy, $\times 1000$ ) from Ad.NGFand Ad. $\beta$ Gal specimens, TUNEL-positive apoptotic cardiomyocytes are pointed by arrows. Graph quantifies apoptosis of cardiomyocytes per $\mathrm{mm}^{2}$ of peri-infarct myocardium section. Values are mean \pm S.E.M. $(n=7){ }^{*} P<0.05$ versus $A d$. $\beta$ Gal

disrupts the action of Foxo-3a and Foxo-1 by phosphorylation, which is a prerequisite for the nuclear exclusion and consequent inactivation of Foxo. ${ }^{14}$ By both immunocytochemistry and western blot analyses of nucleus and cytosolic fractions, we demonstrate that NGF promotes the nuclear accumulation of phospho-Akt and the nuclear exclusion of Foxo-3a and Foxo-1. Notably, we also found that the Akt/Foxo axis can be directly activated by NGF produced by RNCMs, as an autocrine mechanism instrumental to survival. In fact, when we neutralized NGF or blocked its receptor trkA, the levels of both phosphorylated (active) Akt and phosphorylated (inactive) Foxo significantly decreased, while apoptosis increased. Furthermore, apoptosis induced by both $H / R$ and Angll was exacerbated by blocking the endogenous NGF/trkA system. The complex of these findings poses the fundaments for a key role of NGF in cardiac physiology.

In previous publications, we and Cantarella et al. ${ }^{4}$ showed that Akt mediates NGF-induced angiogenesis. ${ }^{3}$ In addition, others published on the prosurvival role of the NGF/Akt axis in neuronal cells. ${ }^{29}$ The antiapoptotic role of Akt in the heart is matter of great attention, as reviewed recently by Catalucci and Condorelli. ${ }^{30}$ Our findings show an additional factor which can impact on this signaling pathway for the control of cardiomyocyte fate.

Apoptosis contributes to cardiac dysfunction in coronary heart disease as well as in idiopathic cardiomyopathy. Not many remedies to combat apoptosis are clinically available. Consequently, the identification of factors able to counteract cardiomyocyte apoptosis is important and may help in establishing new strategies for prevention of heart failure. We reasoned that, since cardiomyocytes release NGF for their own protection, this mechanism could be amplified for therapeutic purposes. Overexpression of NGF led to activation of Akt/Foxo pathway and endowed RNCMs with resistance from hypoxia/reoxygenation or Angll-induced apoptosis, as documented by both immunostaining for activated caspase-3 and TUNEL assay. Supplementation of recombinant NGF to rat adult cardiomyocytes exerted similar protective effects, suggesting that the NT is equally effective in mature cardiac cells.

As a final proof of concept, we aimed to demonstrate the prosurvival action of NGF in vivo. To this aim, we injected the human NGF gene into the rat peri-infarct myocardium, the area that typically shows high levels of cardiomyocyte apoptosis. ${ }^{25-29}$ Results indicate the successful transduction of rat heart with the transgene and, most importantly, the benefit for cardiomyocyte survival in the peri-infarct myocardium. The in vivo cardiac effect of NGF may be attributable to both direct and indirect actions. In fact, similarly to what already seen in limb muscles, ${ }^{3,5} \mathrm{NGF}$ proved to promote myocardial neovascularization (Meloni and Emanueli, unpublished results, 2005), which may account for a better perfusion and improved delivery of oxygen and nutrients to the infarctspared myocytes.

In conclusion, we demonstrated that NGF directly targets the cardiomyocyte and promote its survival. Our discovery 
implicates NGF as a possible therapeutic agent for the cure of degenerative heart disease and also encourages research aimed at unraveling the prosurvival action of other NTs on cardiovascular cells.

\section{Materials and Methods}

RNCM culture. All the procedures involving laboratory animals conform to the Guide for the Care and the Use of Laboratory Animals published by the US National Institute of Health (NIH publication No. 85-23, revised 1996), the guidelines and regulations of the University of Bristol and the United Kingdom Home Office, and of the University of Parma and the Italian Minister of Health. RNCMs were isolated from 2- to 3-day-old Wistar rats (bred at the University of Bristol), as previously described. ${ }^{31}$ Cardiomyocytes were seeded on gelatin-coated plates and maintained in 4:1 DMEM-M199 serum-free medium. The level of purity of RNCM cultures was routinely assessed by staining for the cardiac marker $\alpha$-sarcomeric actin.

Isolation of rat adult cardiomyocytes. Wistar rats (Charles River, Margate, UK) were anesthetized and heparinized and their hearts were rapidly excised and hung in a Langhendorff apparatus. Isolated cells were obtained by collagenase perfusion of the heart, as previously described ${ }^{32}$ Cardiomyocytes were plated on laminin-precoated two-well chamber slides and maintained in M199 serum-free medium.

RNA extraction and RT-PCR. Total RNA of RNCMs was extracted using RNAesy mini kit (Qiagen, Valencia, CA, USA). First-strand cDNA was synthesized from $1 \mu \mathrm{g}$ of total RNA using $200 \mathrm{U}$ of Moloney-murine leukemia virus reverse transcriptase (Invitrogen Ltd, Paisley, UK) and oligo-dT oligomer (Invitrogen) as primer. One microliter of each CDNA preparation was amplified by PCR using the following set of primers: rat NGF- $\beta$, forward, $5^{\prime}$-CTTCAGCATTCCCTTGACAC- $3^{\prime}$ and reverse, $5^{\prime}$-TGAGCACACACACGCAGGC- $3^{\prime}$ (amplified PCR products: NGF- $\beta$, $592 \mathrm{bp}$ ); human NGF- $\beta, 5^{\prime}$-GGCTGCCTGGCGGTTTAT- $3^{\prime}$, and reverse, $5^{\prime}$ GGCAGGTCAGGCTCTTCTCA-3' (primers amplify human NGF CDNA but do not amplify rat NGF cDNA. Amplified PCR products: hNGF- $\beta, 192 \mathrm{bp}$ ), rat trkA, forward, 5' GCATCCTCTTACCGCAAGTT-3', and reverse, 5'-CAGACTCCTAGCCCCAG AACG -3' (amplified PCR products: trkA, 306 bp); 18S rRNA forward, 5' TAGAGGG ACAAGTGGCGTTC-3', and reverse, $5^{\prime}$-TGTACAAAGGGCAGGGACTT-3'.

Western blot analyses. Western blot analyses were carried out as described. ${ }^{3,33}$ The equivalent of $50 \mu \mathrm{g}$ of proteins (with the exception of $30 \mu \mathrm{g}$ of proteins for western blot analyses of nuclear and cytosolic RNCM fractions) was loaded on each lane, resolved by SDS-PAGE and transferred to a nitrocellulose membrane (Amersham Bioscience, Piscataway, NJ, USA). Primary antibodies were for Akt, phospho-Akt (Ser 473), Foxo-1, Foxo-3a (all from Cell Signaling, Danvers, MA, USA; 1 : 1000), phospho-Foxo-1 (Ser256) and phospho-Foxo-3a (Thr32) (both from Upstate, Chandelers, Ford, UK; 1:500), trkA (1:1000, donated by Dr. Dawbarn), phospho trkA (Tyr490) (Calbiochem, San Diego, CA, USA; $1: 500), \alpha / \beta$ tubulin (Cell Signaling 1:1000), V5 epitope tag (Novus Biologicals, Littleton, CO, USA, 1:5000), histone H3 (Lake Placid Biologicals, Lake Placid, NY, USA $1: 5000$ ), and $\alpha$-sarcomeric actin (DAKO, Ely, UK; $1: 500$ ). Secondary antibodies were: anti-rabbit, anti-mouse, or anti-goat IgG-horseradish peroxidase conjugate (Amersham Bioscience; 1:5000).

ELISA for NGF on the RNMCs conditioned medium. RNCMs were maintained in serum-free medium for $24 \mathrm{~h}$ and their conditioned medium was collected and assayed for NGF by ELISA (NGF $E_{\max }^{\mathbb{R}}$ ImmunoAssay Systems, Promega, Southampton, UK).

Differentiation of PC12 cells. PC12 were stimulated with the RNCM conditioned medium for 3 days and their morphology was examined under a phasecontrast microscopy. The number of differentiated PC12 was counted in five randomly select fields. The criterion for distinguishing differentiated from undifferentiated PC12 was neurite outgrowth greater in length than two cell-body diameters.

Fluorescent immunocytochemistry. Cells were cultured in gelatin (RNCMs)- or laminin (rat adult cardiomyocytes)-coated Labtek ${ }^{\mathrm{TM}}$ slides (Nalge Nunc Int., Rochester, NY, USA), fixed with $4 \%$ paraformaldehyde (PFA, $10 \mathrm{~min}$, RT), permeabilized ( $0.2 \%$ Triton $X-100$ in PBS, 20 min, RT), blocked (5\% normal goat serum, $1 \mathrm{~h}, \mathrm{RT}$, and incubated with one of the primary antibodies $\left(16 \mathrm{~h}, 4^{\circ} \mathrm{C}\right)$ for: $\alpha$-sarcomeric actin (DAKO; $1: 50$ ), NGF (Santa Cruz Biotechnology, Santa Cruz, CA, USA; $1: 50$ ), cleaved-caspase-3 (Asp175) (Cell Signaling; 1: 100), Foxo3a, Foxo-1 (Upstate), or phospho-Akt (Ser473) (Cell Signaling 1:100). This was followed by incubation (1 h, RT) with rabbit anti-mouse PE-conjugated secondary antibody (1:200; Sigma-Aldrich, St. Louis, Missouri, USA) or goat anti-rabbit Alexa ${ }^{\circledR}$-Green 488-conjugated secondary antibody $(1: 200$, Molecular Probes, Paisley, UK). In negative controls, the primary antibody was substituted with nonimmune IgG. Coverslips were mounted using Vectashield ${ }^{\circledR}$ (Vector Laboratories, Peterborough, UK) mounting medium containing 4',6 diamidino-2-phenyindole (DAPI) or propidium iodide (PI). Cells were observed under a fluorescence microscope (Olympus BX40, Southall, UK).

Preparation of nuclear/cytosolic fractions of RNCMs. RNCMs $\left(10 \times 10^{6}\right)$ were lysated and cytoplasmic and nuclear extracts were prepared according to the instructions of the NE-PER ${ }^{\circledR}$ nuclear and cytoplasmic extraction kit (Pierce, Rockford, IL, USA).

Preparation of NGF adenovirus. A pCMV-Sport 6 containing the complete cDNA of human NGF $\beta$ ( $p . N G F$ ), cloned into Notl and Sall restriction sites, was purchased (LGC Promochem, Teddington, UK). To prepare an adenoviral vector carrying human NGF- $\beta$ (Ad.NGF), the coding sequence for the NGF- $\beta$ from $p . N G F$ was amplified (KOD proofreading DNA polymerase, Novagen, San Diego, CA, USA) using primers GCTAGCGTAATGTCCATGTTGTTCTAC (Nhel site) and GGATCCTCTCACAGCCTTCCT (BamHI site and stop signal removed) to allow the inclusion of a V5 tag at the $\mathrm{C}$-terminus. The fragment was excised and subcloned in the shuttle vector pDC515 (Microbix Biosystems, Toronto, Canada) modified to contain the V 5 coding sequence. A replication-deficient adenovirus was generated by site-specific FLP-mediated recombination of the cotransfected shuttle and genomic plasmids in 293 cells. Viral stocks were amplified, $\mathrm{CsCl}$ banded, and titrated. $^{31}$

NGF gene transfer to cultured RNCMs. RNCMs were grown in 4:1 DMEM-M199 and were infected overnight with Ad.hNGF or Ad. $\beta$ Gal (each at 50 M.O.I.). After 24 and $48 \mathrm{~h}$, the capacity of $A d . h N G F$ to transduce RNCMs was determined by RT-PCR for human NGF, western blot for the V5 Tag on RNCM lysates or RNCM CCM, and by NGF ELISA (Promega) of the RNCM CCM. In a supplementary experiment, at $24 \mathrm{~h}$ from infection, RNCMs were incubated with Angll $\left(10^{-7} \mathrm{M}\right)$ for $24 \mathrm{~h}$ or submitted to $12 \mathrm{~h}$ hypoxia and $12 \mathrm{~h}$ reoxygenation before being analyzed by RT-PCR for human NGF cDNA level or western blot for V5 (both cell lysated and CCM)

In vitro TUNEL assay of RNCMs. Cells were cultured in gelatin-coated Labtek $^{\mathrm{TM}}$ slides (Nalge Nunc Int.) and fixed with $4 \%$ paraformaldehyde (PFA, $10 \mathrm{~min}, \mathrm{RT})$. Endogenous peroxidase activity was quenched with $3 \% \mathrm{H}_{2} \mathrm{O}_{2}$ in methanol ( $5 \mathrm{~min}, \mathrm{RT}$ ). TUNEL assay was performed according to the manufacture's instruction (Calbiochem). Nuclei were counterstained with methyl green dye.

\section{Hypoxia/reoxygenation and Angll apoptosis assays on} RNCMs. Following infection with Ad.NGF or Ad. $\beta$ Gal, apoptosis of serum-freecultured RNCMs was induced by two methods: (1) cells were transferred to an anaerobic chamber which was purged with $95 \% \mathrm{~N}_{2} / 5 \% \mathrm{CO}_{2}$. RNCMs were kept under hypoxia for $12 \mathrm{~h}$ before being reoxygenated under normoxic conditions for $24 \mathrm{~h}$. Incubations were performed at $37^{\circ} \mathrm{C}^{19}$ or (2) cells were incubated with Angll $\left(10^{-7} \mathrm{M}\right)$ for $24 \mathrm{~h} .{ }^{20}$ Apoptosis was detected by both cleaved caspase 3 staining and TUNEL assay. At least 800 RNCMs (in eight randomly chosen $\times 400$ fields from three independent experiments) were scored in a blinded fashion to calculate the percentage of cleaved caspase 3-positive or TUNEL-positive nuclei. Data are expressed as the average percentage of apoptotic cells or nuclei.

Apoptosis assays in the presence of K252a, LY294002, Ad.DN-Akt, or Ad.AAA Foxo-3a. Adenoviruses carrying a dominant negative form of Akt $(A d . D N-A k t)^{3}$ and a constitutive active mutant form of Foxo-3a which has Alanine residues in the three phosphorylation sites of Akt and is consequently resistant to Akt phosphorylation (Ad.AAAFoxo3a) (Vector Biolabs, Philadelphia, PA, USA) were used for this experiment. RNCMs were coinfected with Ad.NGF or $A d . \beta G a l$ (50 m.o.i) plus $A d . D N-A k t, A d . A A A F o x 03 a$, or $A d$. $\beta G a l$ (the latter as control of either Ad.DN-Akt or Ad.AAAFoxo3a) (100 M.O.I.). After $24 \mathrm{~h}$ from infection, serum-freecultured RNCMs underwent $5 \mathrm{~h}$ hypoxia/ $24 \mathrm{~h}$ reoxygenation. In separate 
experiments, RNCMs were infected with Ad.NGFor Ad. $\beta G$ al and, after $24 \mathrm{~h}$, treated with the PI3K inhibitor LY294002 (Sigma-Aldrich, $50 \mu \mathrm{M}$ ), the trkA inhibitor K252a (Sigma-Aldrich, $100 \mathrm{nM})$, or their vehicle $(0.1 \%$ DMSO) starting $30 \mathrm{~min}$ before undergoing $5 \mathrm{~h}$ hypoxia/ $24 \mathrm{~h}$ reoxygenation. Apoptosis was evaluated by both cleaved caspase-3 staining and by TUNEL assay. Data are expressed as the average percentage of apoptotic myocytes and nuclei, respectively. For reference, additionally experiments were performed treating RNCMs as described above, but maintaining them under normoxia.

RNCM transfection with siRNA and apoptosis assay. RNCM were plated on gelatin-coated 12-well plate and transfected (using Oligofectamine ${ }^{\mathrm{TM}}$, Invitrogen) with Akt siRNA (50 nM, SignalSilence ${ }^{\circledR}$ Akt siRNA, \# 6211 Cell Signaling), which targets both Akt1 and Akt2, or negative control scramble siRNA. Knockdown of Akt protein at $48 \mathrm{~h}$ following Akt siRNA transfection was confirmed by western blotting. Following transfection, RNMCs were infected with Ad.NGF or Ad. $\beta$ Gal ( 50 m.o.i each). After $24 \mathrm{~h}$ from infection, RNCMs underwent $5 \mathrm{~h}$ hypoxia/ $24 \mathrm{~h}$ reoxygenation or were maintained under normoxia (for $29 \mathrm{~h}$ ). Apoptosis was evaluated by both cleaved-caspase 3 and TUNEL assay, as described above.

In vitro TUNEL assay of rat adult myocytes. Cells were cultured in laminin-coated Labtek ${ }^{\mathrm{TM}}$ slides (Nalge Nunc Int.) and fixed with $4 \%$ paraformaldehyde (PFA, $10 \mathrm{~min}, \mathrm{RT}$ ). TUNEL assay was performed according to the manufacture's instruction (Calbiochem). Then, the same sections were stained with a mouse monoclonal primary antibody for $\alpha$-sarcomeric actin (Sigma, 1:30, $\left.16 \mathrm{~h}, 4^{\circ} \mathrm{C}\right)$ followed by secondary antibody rabbit anti-mouse TRITC $(1: 20,1 \mathrm{~h}, \mathrm{RT}$, Sigma). Nuclei were counterstained with DAPI (Sigma).

$H / R$ and Angll apoptosis assays on rat adult cardiomyocytes. $H / R$ (6 $\mathrm{h}$ hypoxia, $18 \mathrm{~h}$ reoxygenation) was performed accordingly to Kang et al. ${ }^{24}$ Apoptosis was detected by both cleaved-caspase 3 staining and TUNEL assay. Cardiomyocytes were stained by an antibody for $\alpha$-sarcomeric actin. Cells were examined at $\times 400$ magnification. Cleaved-caspase-3-positive or TUNEL-positive cells were scored in a blinded fashion in eight randomly chosen fields from three independent experiments. Data are expressed as the average percentage of apoptotic cells. Angll apoptosis assay was performed as previously reported. ${ }^{23}$ Isolated rat adult cardiac myocytes were treated with Angll $\left(10^{-9} \mathrm{M}\right)$ in the presence of recombinant NGF $(50 \mathrm{ng} / \mathrm{ml})$ or PBS for $24 \mathrm{~h}$. Also in this case, apoptosis was evaluated by both cleaved-caspase 3 staining and TUNEL assay.

Rat myocardial infarct and intra-myocardial NGF gene transfer. Wistar rats (Charles River, Calco, Italy) were anesthetized by ketamine chloride $(40 \mathrm{mg} / \mathrm{kg}$ i.p., Merial, Milan, Italy) plus medetomidine hydrochloride $(0.15 \mathrm{mg} / \mathrm{kg}$ i.p., Pfizer Italia srl, Latina, Italy) and artificially ventilated. Myocardial infarct was induced by permanent ligature of the left coronary artery. After $3 \mathrm{~min}$ of coronary ligation, Ad.NGF or Ad. $\beta$ Gal (each at $10^{8}$ p.f.u.) was injected into four adjacent points of the Ml border zone. At 3 days postsurgery, the heart of anaesthetized rats ( $n=3$ per group) was harvested and processed for RNA extraction. Other rats ( $n=7$ per group) were killed at 7 days post-Ml by arresting the heart by intraventricular injection of cadmium chloride. Hearts were perfusion/fixed with buffered formalin and embedded in paraffin. Heart sections were submitted to V 5 immunohistochemistry and TUNEL apoptosis assay (vide infra).

V5 immunohistochemistry of heart sections. Heart sections were incubated with rabbit serum to block aspecific binding and then with a goat-raised primary antibody for V5 $\left(1: 100,16 \mathrm{~h}, 4^{\circ} \mathrm{C}\right.$, Novus Biologicals), followed by a secondary antibody rabbit anti-goat FITC $\left(1: 20,1 \mathrm{~h}, 37^{\circ} \mathrm{C}\right.$, Sigma). Then, sections were blocked with rabbit serum and stained with $\alpha$-sarcomeric actin (monoclonal mouse, $1: 30,16 \mathrm{~h}, 4^{\circ} \mathrm{C}$, Sigma), followed by secondary antibody rabbit anti-mouse TRITC $\left(1: 20,1 \mathrm{~h}, 37^{\circ} \mathrm{C}\right.$, Sigma). Nuclei were counterstained with DAPI (Sigma). Sections were analyzed with a fluorescent microscope.

TUNEL assay on rat heart sections. Sections $(4 \mu \mathrm{m})$ of rat hearts were cut and mounted on slides. Following treatment of slides with xylene, graded alcohol, and $\mathrm{H}_{2} \mathrm{O}$, endogenous peroxidase activity was quenched with $3 \% \mathrm{H}_{2} \mathrm{O}_{2}$ in methanol ( $30 \mathrm{~min}, \mathrm{RT}$ ). TUNEL assay was performed according to the manufacture's instruction (Calbiochem). The same sections were then stained with a mouse monoclonal primary antibody for the myocyte marker $\alpha$-sarcomeric actin (Sigma, $1: 30,16 \mathrm{~h}, 4^{\circ} \mathrm{C}$ ), which was revealed by VIP kit (Vector Laboratories).
The numbers of TUNEL-positive apoptotic cardiomyocytes in the peri-infarct myocardium was counted with the aid of ocular reticle, at $\times 1000$ magnification The density of apoptotic cardiomyocytes per $\mathrm{mm}^{2}$ of tissue section was calculated. $^{32,33}$

Statistics. Values are presented as mean \pm S.E.M. Analyses were performed using the SigmaStat 3.1 software. Statistical significance was evaluated by use of unpaired $t$-test for comparison between two groups. For comparison among more than two groups, ANOVA was used, followed by unpaired $t$-test. A $P$-value $<0.05$ was interpreted to denote statistical significance.

Acknowledgements. Dr. Emanueli holds a British Heart Foundation (BHF) Basic Science Lectureship. Andrea Caporali is a BHF PhD student. This study was entirely supported by the BHF. We thank Miss Jill Tarlton (Bristol Heart Institute) for her help in Ad.NGF preparation, Dr. Dawbarn (University of Bristol) for providing trkA antibody and PC12, Dr. Aloe (CNR, Rome) for providing Ab-NGF, Mauro Siragusa (University of Bristol) for helping with the preparation of fluorescent pictures, and Dr. Costanza Lagrasta (University of Parma) for collaborating at the in vivo experiment. The Bristol Heart Institute is a member of the European Vascular Genomic Network of Excellence (EVGN, funded by the European Community).

1. Levi-Montalcini R. The nerve growth factor: thirty-five years later. EMBO J 1987; 6 : $1145-1154$.

2. Chao MV, Rajagopal R, Lee FS. Neurotrophin signalling in health and disease. Clin Sci (Lond) 2006; 110: 167-173.

3. Emanueli C, Salis MB, Pinna A, Graiani G, Manni L, Madeddu P. Nerve growth factor promotes angiogenesis and arteriogenesis in ischemic hindlimbs. Circulation 2002; 106 2257-2262.

4. Cantarella G, Lempeur L, Presta M, Ribatti D, Lombardo G, Lazarovici $P$ et al. Nerve growth factor-endothelial cell interaction leads to angiogenesis in vitro and in vivo. FASEB J 2002; 16: 1307-1309.

5. Salis MB, Graiani G, Desortes E, Caldwell RB, Madeddu P, Emanueli C. Nerve growth factor supplementation reverses the impairment, induced by type 1 diabetes, of hindlimb post-ischaemic recovery in mice. Diabetologia 2004; 47: 1055-1063.

6. Graiani G, Emanueli C, Desortes E, Van Linthout S, Pinna A, Figueroa CD et al. Nerve growth factor promotes reparative angiogenesis and inhibits endothelial apoptosis in cutaneous wounds of type 1 diabetic mice. Diabetologia 2004; 47: 1047-1054.

7. Kermani $P$, Rafii D, Jin DK, Whitlock P, Schaffer W, Chiang A et al. Neurotrophins promote revascularization by local recruitment of TrkB+ endothelial cells and systemic mobilization of hematopoietic progenitors. J Clin Invest 2004; 115: 653-663

8. Kaye DM, Vaddadi G, Gruskin SL, Du XL, Esler MD. Reduced myocardial nerve growth factor expression in human and experimental heart failure. Circ Res 2000; 86: 80-84.

9. leda M, Fukuda K, Hisaka Y, Kimura K, Kawaguchi H, Fujita J et al. Endothelin-1 regulates cardiac sympathetic innervation in the rodent heart by controlling nerve growth factor expression. J Clin Invest 2004; 113: 876-884.

10. Hiltunen JO, Laurikainen A, Väkevä A, Meri S, Saarma M. Nerve growth factor and brainderived neurotrophic factor mRNAs are regulated in distinct cell populations of rat heart after ischaemia and reperfusion. J Pathol 2001; 194: 247-253.

11. Scarisbrick IA, Jones EG, Isackson PJ. Coexpression of mRNAs for NGF, BDNF, and NT-3 in the cardiovascular system of the pre- and postnatal rat. $J$ Neurosience 1993; 13: 875-893.

12. Gan L, Zheng W, Chabot JG, Unterman TG, Quiron R. Nuclear/cytoplasmic shuttling of the transcription factor FoxO1 is regulated by neurotrophic factors. J Neurochem 2005; 93 $1209-1219$

13. Gilley J, Coffer PJ, Ham J. FOXO transcription factors directly activate bim gene expression and promote apoptosis in sympathetic neurons. J Cell Biol 2003; 162: 613-622.

14. Vogt PK, Jiang H, Aoki M. Triple layer control: phosphorylation, acetylation and ubiquitination of FOXO proteins. Cell Cycle 2005; 4: 908-913.

15. Modur V, Nagarajan R, Evers BM, Milbrandt J. FOXO proteins regulate tumor necrosis factor-related apoptosis inducing ligand expression. Implications for PTEN mutation in prostate cancer. J Biol Chem 2002; 277: 47928-47937.

16. Sunters A, de Mattos SF, Stahl M, Brosens JJ, Zoumpoulidou G, Saunders CA et al. FoxO3a transcriptional regulation of bim controls apoptosis in paclitaxel-treated breast cancer cell lines. J Biol Chem 2003; 278: 49795-49805.

17. Skurk C, Izumiya $Y$, Maatz $H$, Razeghi $P$, Shiojima I, Sandri M et al. The FOXO3a transcription factor regulates cardiac myocyte size downstream of AKT signaling. J Biol Chem 2005; 280: 20814-22023.

18. Morris JB, Kenney B, Huynh H, Woodcock EA. Regulation of the proapoptotic factor FOXO1 (FKHR) in cardiomyocytes by growth factors and alpha1-adrenergic agonists. Endocrinology 2005; 146: 4370-4376. 
19. Yin $\mathrm{H}$, Chao $\mathrm{L}$, Chao J. Kallikrein/kinin protects against myocardial apoptosis afte ischemia/reperfusion via Akt-glycogen synthase kinase-3 and Akt-Bad.14-3-3 signaling pathways. J Biol Chem 2005; 280: 8022-8030.

20. Goldenberg I, Grossman E, Jacobson KA, Shneyvays VV, Shainberg A. Angiotensin II-induced apoptosis in rat cardiomyocyte culture: a possible role of AT1 and AT2 receptors. J Hypertension 2001; 19: 1681-1689.

21. Ross AH, McKinnon CA, Daou MC, Ratliff K, Wolf DE. Differential biological effects of K252a kinase inhibitors are related to membrane solubility but not permeability. J Neurochem 1995; 65: 2748-2756.

22. Thornberry NA, Lazebnik Y. Caspases: enemies within. Science 1998; 281: 1312-1316. Review.

23. Kajstura J, Cigola E, Malhotra A, Li P, Cheng W, Meggs LG et al. Angiotensin II induces apoptosis of adult ventricular myocytes in vitro. J Mol Cell Cardiol 1997; 29: 859-870.

24. Kang PM, Haunstetter A, Aoki H, Usheva A, Izumo S. Morphological and molecular characterization of adult cardiomyocyte apoptosis during hypoxia and reoxygenation. Circ Res 2000; 87: 118-125.

25. Olivetti G, Quaini F, Sala R, Lagrasta C, Corradi D, Bonacina E et al. Acute myocardia infarction in humans is associated with activation of programmed myocyte cell death in the surviving portion of the heart. J Mol Cell Cardiol 1996; 28: 2005-2016.
26. Saraste A, Pulkki K, Kallajoki M, Henriksen K, Parvinen M, Voipio-Pulkki LM. Apoptosis in human acute myocardial infarction. Circulation 1997; 95: 320-323.

27. Colucci WS. Molecular and cellular mechanisms of myocardial failure. Am J Cardiol 1997; 80: $15-25$.

28. Takemura G, Fujiwara $\mathrm{H}$. Role of apoptosis in remodeling after myocardial infarction. Pharmacol Ther 2004; 104: 1-16.

29. Ahn J-Y, Liu X, Liu Z, Pereira L, Cheng D, Peng J et al. Nuclear Akt associates with PKCphosphorylated Ebp1, preventing DNA fragmentation by inhibition of caspase-activated DNase. EMBO J. 2006; 25: 2083-2095.

30. Catalucci D, Condorelli GL. Effects of Akt on cardiac myocytes: location counts. Circ Res 2006; 99: 339-341.

31. Sala-Newby G, Freeman NVE, Curto MA, Newby AC. Metabolic and functional consequences of cytosolic 5-nucleotidase-IA overexpression in neonatal rat cardiomyocytes. Am J Physiol Heart Circ Physiol 2003; 285: 991-998.

32. Graiani G, Lagrasta C, Migliaccio E, Spillmann F, Meloni M, Madeddu P et al. Genetic deletion of the p66Shc adaptor protein protects from angiotensin II-induced myocardial damage. Hypertension 2005; 46: 433-440.

33. Spillmann F, Graiani G, Van Lintout S, Meloni M, Campesi I, Lagrasta C et al. Regional and global protective effects of tissue kallikrein gene delivery to the peri-infarct myocardium. Regenerative Med 2006; 1: 235-254.

Supplementary Information accompanies the paper on Cell Death and Differentiation website (http://www.nature.com/cdd) 